\title{
Refractory Mediastinal Lymphoma
}

National Cancer Institute

\section{Source}

National Cancer Institute. Refractory Mediastinal Lymphoma. NCI Thesaurus. Code C153177.

Mediastinal lymphoma that is resistant to treatment. 